\title{
DIOS Y ESTRÉS: ENMIENDAS A LA CREACIÓN. RELIGIÓN EN JOHN STUART MILL ${ }^{1}$ GOD AND STRESS: AMENDMENTS TO CREATION. RELIGION IN JOHN STUART MILL
}

\section{Diego A. Soto Morera \\ Escuela Ecuménica de Ciencias de la Religión}

\begin{abstract}
Resumen
En este trabajo se analiza el concepto de religión en John Stuart Mill como parte de sus Principios de Política Económica. De acuerdo con Mill, no es la muerte de Dios aquello que debe esperarse o propiciarse en una sociedad liberal, pues la religión no está fundada en el miedo. En cambio, el mito fundamental del liberalismo sería el surgimiento de una comunidad del estrés, pues la religión está fundada en el deseo de enmendar la creación y la erradicación del mal. Esta perspectiva será analiza en relación con la tradición de la teología económica cristiana, en la cual, los dones dados por Dios a la humanidad deben ser proliferados, así como una prefiguración del lugar que ocuparía la religión en el mundo moderno según lo imaginaron los textos de Mill.
\end{abstract}

Palabras clave: religión, liberalismo, energía, rendimiento, mill.

\begin{abstract}
This paper analyses the concept of religion in John Stuart Mill as part of his Principles of Political Economy. According to Mill, it is not the dead of God what is to be expected or propitiated in a liberal society, for religion is not anchored in fear. Instead, the fundamental myth of liberalism would be the rise of a fellowshipof stress, where God requires the partnership of humans, for religion is anchored in desire to amend the creation and the eradication of evil. This perspective will be analyzed in relation with the tradition of Christian theological economy, in which, God's gifts to mankind must be proliferated, and as prefiguration of the role of religion in the modern world imagined by Mill's texts.
\end{abstract}

Keywords: Religion. Liberalism. Energy. Performance. Mill.

1 Este manuscrito es producto del proyecto de investigación Hacia una Economía Política de la Carne en Costa Rica. Etapa Segunda, de la Escuela Ecuménica de Ciencias de la Religión, Universidad Nacional de Costa Rica. 


\section{Introducción}

Este artículo estudia el concepto de religión al interior de la economía política de John Stuart Mill. Lo religioso, en la perspectiva del filósofo inglés, no se advirtió como residuo incómodo de la infancia humana, un cadáver sagrado, destinado al rincón más privado de su existencia. Mill prefiguró que lo religioso tendría continuidad al interior del liberalismo, no como herramienta de control moral, sino como expresión de la disposición económica del ser humano, por la cual se le requiere enmendar su estado. La tesis básica de este artículo será mostrar cómo en Mill, el mito fundamental del liberalismo no es la muerte de dios, sino la alianza del estrés, donde el problema básico de la existencia es el aumento del rendimiento de lo creado.

En la obra de J. S. Mill resulta evidente que el imperativo económico, según el cual, cada uno debe realizar una apropiada administración de su vitalidad-energía, no remite exclusivamente a un criterio técnico de productividad. En una discusión mucho más amplia, el filósofo inglés lo ubica como la forma en la cual cada ser humano responde a la exigencia antropológica que nos define como especie. Según su perspectiva, la creación y sus criaturas no deben solo conservarse en su integridad, sino que, tal como se predica de la parábola de los talentos, lo creado debe ser enmendado y ensanchado, debe llevarse hasta su grado más pleno, a tal punto que finalmente el bien se imponga sobre el mal. El problema industrial de captura eficiente de vitalidad se inscribe dentro de una consideración de mayor amplitud en torno a la naturaleza, la divinidad y sus propósitos, donde cada elemento en el universo porta el requerimiento de alcanzar un estado de mayor perfección.

Los ensayos sobre la religión de J. S. Mill se ocupan de esta cuestión. En el trasfondo de la discusión liberal sobre el progreso yace una consideración acerca de la naturaleza y el ser humano, donde el filósofo inglés aborda con detenimiento los problemas legados por siglos de tradición teológica cristiana, no como un recurso didáctico o meramente histórico, sino como una forma de ahondar el problema básico de la economía teológica con respecto al tipo de administración de sí que logra captar (en su amplia acepción de producir y encauzar) el máximo potencial de lo creado. En su consideración sobre la religión, Mill no solo se separa de muchas de las posturas clásicas (muy enraizadas aún) sobre el utilitarismo de la religión para la sociedad liberal, sino que muestra cómo el problema de la captura de energía-vital debe ser abordado desde su profundidad antropológica. 
Finalmente, el propósito de estas consideraciones será mostrar cómo una preocupación básica de la economía política liberal, a saber, la captura de vitalidad-energía, no se desplegó contra el acervo teológico de la tradición cristiana europea, como se predica de un discurso sobre la mayoría de edad secularizada de la modernidad. Tampoco el liberalismo se limitó a citar la tradición teológica como recurso para mediar o asegurar la aceptación popular de la nueva corriente cultural. Las discusiones en torno a la captura de vitalidad-energía se dieron a partir del influjo cultural de la tradición de la economía teológica, cuyo material gramatical permitió articular y ahondar los modos de comprensión sobre la producción de excesos y su vínculo con el desarrollo de la maximización de la labor industrial.

Establecer que la tradición de teología económica ofrece los trasfondos culturales que permitieron pensar los aparatos de captura de vitalidad para la economía política liberal supone evitar simplificaciones. En primer lugar, sugerir que las formas teológico-religiosas de un momento histórico deben ser analizadas en sus asociaciones con la economía política, no implica determinar las primeras, en un nivel ideológico, como aquella supraestructura que despliega una distorsión farmacológica de la realidad. Asimismo, a partir de un espíritu funcionalista, delimitar los servicios morales que instituciones religiosas brindan en un momento particular al tipo de emprendimientos requeridos por el liberalismo, no agota los traslapes entre la economía política y la tradición teológica cristiana. En cada uno de los casos aludidos, se reduce el aparato de captura teológico a modos, aunque diversos, de aprisionamiento, sin ocuparse de una locación más estructural de lo teológico con respecto a la economía política, donde podemos advertir su vínculo con la formación de excedentes, y su incorporación, como procesos constitutivos de la empresa liberal.

En adelante, procuraré mostrar cómo la economía política liberal, al menos, según la presentación de uno de sus pensadores más representativos del siglo XIX, no establece una ruptura definitiva con la tradición de teología económica cristiana, ni tampoco se limita a usufructuar beneficios particulares de instituciones religiosas. El propósito consiste en ubicar los vestigios de un alcance mucho más constitutivo de la tradición teológica al interior de la formulación de los ejes fundamentales del liberalismo decimonónico, a saber, ofrecer las coordenadas básicas que enmarcaron la captura de la vida. De ahí que nuestra fuente de discusión central proviene, en este apartado, no de textos cristianos o tratados teológicos, sino de autores que abordaron problemáticas básicas vinculadas a la 
industria decimonónica. Los textos de J. S. Mill serán analizados en esta clave, con el propósito de considerar los trasfondos teológicos a partir de los cuales pensó una economía política de tipo liberal con base en una consideración sobre la naturaleza.

\section{Energía industrial: deseo y productividad nacional en John Stuart Mill}

Al interior de las distintas discusiones presentes en su Principles of Political Economy (1848), texto básico en la formación del pensamiento económico de su época, John Stuart Mill dedica un apartado al análisis de las causas de la superioridad productiva de algunas naciones, particularmente, en lo que se refiere a la energía laboral: ¿por qué algunas naciones, se preguntó Mill, cuentan con un mayor acervo de energía laboral para su aprovechamiento y empleo? (Mill 102-106).

Para explicar las diferencias de capacidad energética laboral entre las naciones, Mill hace referencia a un principio etnográfico de su tiempo: en lugares «in which mere existence is a pleasure» la energía corporal, si bien abundante, es gobernada por las pasiones y se desvía de toda actividad disciplinada. Son más bien las dificultades del medio hostil "that nourish bodily and mental energy», razón por la cual, ha sido en la premura de las adversidades del entorno, «Up
North», donde la empresa conquistadora, el vigor militar, el pensamiento especulativo y la energía industrial se han desarrollado y consumado con mayor alcance, efectividad y propiedad. ${ }^{2}$ Mill no concibe esta particularidad etnográfica, asociada con las circunstancias climatológicas y geográficas, como una constante natural de la condición humana, la cual vincularía, por algún capricho ontológico, raza y energía corporal. Mill asume esta diferencia como una variable antropológica, por lo tanto, susceptible de transformación.

El despliegue de la energía laboral requiere de formas específicas de

2 Se trata de una concepción que compartía, siglos antes, un autor como Jean Jacques Rousseau, cuando intenta explicar las bases antropológicas que explican las diferencias entre las lenguas de países cálidos y septentrionales: "En aquellos horribles climas donde todo está muerto durante nueve meses del año, donde el sol solo calienta el aire algunas semanas para enseñarles mejor a los habitantes de qué bienes están privados y prolongar así su miseria, en esos lugares donde la tierra no da nada si no es a fuerza de trabajo, y donde la fuente de la vida parece estar más en los brazos que en el corazón, los hombres, atareados sin cesar buscando provisiones para su subsistencia, se preocupan apenas por lazos más dulces [...] La ociosidad que nutre las pasiones cedió el lugar al trabajo que las reprime: antes de preocuparse por vivir feliz, era preciso preocuparse por vivir [...] Los de los países cálidos tienen pasiones voluptuosas, relacionadas con el amor y la suavidad: la naturaleza hace tanto por los habitantes que estos casi no tienen nada que hacer; si un asiático tiene mujeres y reposo, está contento. Pero en el Norte, cuyos hombres consumen mucho sobre un suelo ingrato, los hombres sometidos a tantas necesidades son fácilmente irritables; les inquieta todo lo que se hace a su alrededor" (Rousseau 55-56). 
trabajo: «Labour which relates to human beings». Seres humanos, aun aquellos que Mill considera salvajes y, por lo tanto, acostumbrados a las condiciones más favorables (Mill refiere a «the negroes of Jamaica and Demerara»), pueden ser educados de modo que puedan reapropiarse de su energía industrial: "To civilize a savage, he must be inspired with new wants and desires, event if not of a very elevated kind, provided that their gratification can be a motive to steady and regular bodily and mental exertion" (Mill 104). El salvaje, cuya energía corporal es abundante pero distribuida de manera amorfa, puede ser civilizado, lo que quiere decir, enseñado a concentrar y canalizar su «industrial energy» (aquella que constantemente está al servicio del trabajo). Para esto se requiere de una pedagogía que pivota sobre la maleabilidad del deseo humano. Por efectos de sublimación del deseo, la energía corporal puede ser reencauzada, de modo que sirva a los propósitos de una disciplina laboral, sobre la cual descansa toda posibilidad de maximización de la producción.

Mill tiene claro que la energía corporal fluye, y que los deseos participan de la captación y canalización de este flujo. El aprovechamiento industrial de la energía corporal, incluso en lossalvajes de las regiones cálidas, no pasa por una proscripción del placer. El deseo funciona, según Mill, como un vector energético, susceptible de modificación en su variable direccional. Nuevos deseos pueden provocarse, «event if not of a very elevated kind» (una referencia a David Hume, quien señaló la necesidad y beneficios de desarrollar the delicacy of taste sobre la delicadeza de las pasiones) (Hume 3-8). De esto se sigue que, con el debido trabajo, es posible variar la orientación de flujo energético, así como su intensidad. La captación, como redefinición del curso del deseo, permite su inscripción, por coincidencia o correlato de destinos gratificantes, con las metas de emprendimientos industriales. La energía corporal, que no cesa de fluir en condiciones óptimas (saludables), no debe ser reprimida, restringida, menos aún sometida y obligada. La tesis que sostiene Mill es que una política económica debe atender a los destinos del deseo, de modo que se puedan identificar, promover e intensificar aquellos que potencian, o al menos soportan el despliegue de la energía industrial.

Con mucha antelación a Freud, una variable básica de la economía política liberal era el lugar de la sublimación del deseo y sus vínculos con la economía política. Alrededor de ochenta años más tarde, en su texto El malestar de la cultura (1930), el vienés se refirió al vínculo constitutivo entre economía política y economía libidinal (Freud 109-110). El padre del psicoanálisis puso en tela de juicio aquella 
tesis «comunista», según la cual, de la anulación de la propiedad privada se sigue la redención del mal. Si bien Freud se excusó de abordar la teoría económica que sustenta esta tesis ( «No es de mi incumbencia la crítica económica al sistema comunista»-dijo-), criticó la fragilidad de sus premisas psicológicas.

Según el psicoanalista la propiedad privada, pilar fundamental de la economía política liberal, se articula y extiende en la economía libidinal de los sujetos, donde encuentra soportes y apoyos. No se debe a que la propiedad privada sea un dato psicológico o una condición ontogenética, pues ninguna pulsión «ha sido creada por la institución de la propiedad». La tesis de fondo postula la profunda interdependencia entre economía política y economía libidinal, en términos de coincidencia de sus destinos pulsionales. No es posible desplegar un proyecto económico sin un trabajo sobre la economía libidinal que cimiente las bases psicológicas del modelo productivo requerido. La «ilusión» de fondo, según Freud, es que de la eliminación de la propiedad privada se siga la cancelación de la economía libidinal a la que está vinculada, lo que es un absurdo debido a su profunda imbricación histórica. Según la discusión anterior, J. S. Mill (y toda una larga tradición británica) tenía muy claro este principio, muchas décadas antes de su formulación en el lenguaje psicoanalítico.
Un principio básico en la economía política de John Stuart Mill es la necesidad de administrar el deseo humano como factor de la productividad nacional. Esto se sigue del vínculo que advertía entre la maleabilidad del deseo y el acervo de la energía corporal dispuesta para la industria. El tesoro nacional se cuenta también por la calidad de las fibras internas de los cuerpos individuales, pero, principalmente, por un meticuloso trabajo sobre esos cuerpos, capaz de movilizar (no reprimir) deseos y gratificaciones según las coordenadas y magnitudes requeridas por la maximización productiva.

El Estado debe tener presente que la administración apropiada de los nervios, músculos y deseos es un componente básico del desarrollo de la productividad nacional, en tanto asegura la eficiencia energética requerida por la industria. La economía política del siglo XIX señaló la necesidad de ocuparse de la moral de la población como garantía del acervo de energía laboral requerido para la producción: "The moral qualities of the labourers are fully as important to the efficiency and worth of their labour, as the intellectual" (Mill 109). La deficiencia moral, ${ }^{3}$

3 Previamente, Adam Smith ha señalado: "That the tendency of virtue to promote, and of vice to disturb the order of society, when consider it coolly and philosophically, reflects a very great beauty upon the one, and a very great deformity upon the other, cannot, as I have observed upon a former occasion, be called in question" (Smith, 
como en el salvaje, hace que la energía corporal se disperse de modo amorfo, pero, por otro lado, el rigorismo moral puede producir cuerpos enervados, incapaces de movilizar sus deseos y gustos según el proyecto requerido por la productividad nacional.

De esta forma, no es la represión del cuerpo individual, inicialmente tampoco el disciplinamiento de los miembros por parte de una institución, lo que interesa a la economía política en la formulación de John Stuart Mill. El núcleo básico no es el cuerpo como una unidad sólida de falanges, móviles pero invariables, susceptibles de adiestramiento no de transformación. Existe una preocupación económica previa al disciplinamiento molar de los miembros. Hay un flujo en la corporalidad misma, que Mill denomina energía corporal, una vitalidad orgánica que debe ser captada, no solo para usufructuar de su dinamismo a nivel industrial, sino para enriquecerla. La captura de esta vitalidad, según estos principios de economía política, no se despliega mediante mecanismos de confinamiento que la recluyen y, por lo mismo, la exteriorizan. No es una forma de agente patógeno que debe ser apresado o incluso descartado y desechado, aunque

316). Para Smith el cultivo de la virtud, y la correlativa erradicación del vicio, permiten que la gran máquina que es la sociedad humana funcione de manera perfecta y bella. ciertamente hay peligros asociados a su indebida administración que competen a la producción y a la salud del cuerpo social. La captura que interesa a Mill sobre esta vitalidad se comprende en términos de afluencias que reconfiguran corrientes de flujo en intensidad, cauce, caudal y alcance.

Asimismo, las lógicas de captación que piensa John Stuart Mill no se definen como una actividad centralizada en el Estado, el cual desplegaría, desde arriba, mediante instituciones o aparatos gubernamentales, esquemas uniformes que aseguren el correcto devenir del flujo corporal. Por el contrario, para Mill, cada individuo, luego de una serie de trabajos que posibilita y desarrolla la vida social (principalmente mediante la educación), debe potenciar las cualidades que le permitan administrar en forma eficiente su propia vitalidad, desarrollar sus capacidades individuales, y mediante esta práctica de sí mismo, contribuir al común de su «raza», y al beneficio del producto nacional:

It is not by wearing down into uniformity all that is individual in themselves, but by cultivating it and calling it forth, within the limits imposed by the rights and interest of others, that the human beings become a noble and beautiful object of contemplation; and as the works partake the character of those who do them, by the same process 
human life become rich, diversified, and animating, furnishing more abundant aliment to high thoughts and elevating feelings, and strengthening the tie which binds every individual to the race, by making the race infinitely better belonging to. In proportion to the development of his individuality, each person becomes valuable to others. There is a greater fullness of life about his own existence, and when there is more life in the units there is more in the mass which is composed of them (Mill, "On Liberty", 266).

La belleza del ser humano se propicia ahí donde es capaz de administrar apropiadamente su vitalidad («human life») para fortalecerla, mejorarla, diversificarla (no uniformizarla): "Each is the proper guardian of his own health, whether bodily, mental and spiritual" (Mill, "On Liberty", 226). Un trabajo apropiado de administración de la propia vitalidad está lejos de ser una actividad egoísta, pues, cada individuo que se ocupa de fortalecer su propia vida contribuye positivamente al fortalecimiento del conjunto: «when there is more life in the units there is more in them as which is composed of them». Fortalecer la vitalidad individual, ocuparse de sí mismo, es la mayor contribución que cada ser humano brinda a la raza, al cuerpo social, al embellecimiento de la nación.

La administración de la vitalidad ocupa, según la economía liberal, un lugar fundamental al interior del proyecto nacional. Las actividades sobre las cuales descansa la industria y producción nacional pasan por la capacidad de los individuos de desplegar una apropiada administración de sí mismos. Por ejemplo, aspectos tan sensibles para la economía nacional, tales como la adecuada regulación del «desire of acumulation», dependen del desarrollo de una propicia gestión que los individuos hacen de sí mismos. Este factor contribuye a explicar, según Mill, la razón por la cual una sociedad como la China, si bien superior a nivel asiático, es inferior con respecto a la europea en términos de incremento del capital: "From various circumstances in their personal habits and social conditions, it might be anticipated that they [the Chinese] would possess a degree of prudence and self-control greater than other Asiatics, but inferior to most European nations" (Mill, "Principles of Political Economy”, 167). El self-control, o bien, la administración de sí, es una variable importante de la economía política liberal, en tanto afecta aspectos tan sensibles como la productividad, así como la acumulación e incremento del capital.

De esto se sigue que, según la economía política del siglo XIX, las deficiencias en la productividad no dependen exclusivamente de aspectos técnicos (como la tecnificación de los procesos de producción), sino también 
de la eficiente educación que un gobierno pueda implementar al interior de una nación en lo que respecta al self-control. Por ejemplo, en naciones donde la variable tecnológica es similar, un pobre desarrollo de la capacidad individual-social de providencia (que Mill vincula «with intelectual as well as moral causes») impacta negativamente la productividad nacional: "That it is a defect of providence, not defect of industry, that limits production among the Chinese, is still more obvious than in the case of the semi-agriculturized Indians" (Mill, "Principles of Political Economy", 168). Esta capacidad de autocontrol, o administración de sí, es lo que permite formas de captación de vitalidad adecuadas a prácticas eficientes de incremento productivo de capital. El lugar que ocupa esta variable en la explicación de Mill resulta tan central, que le permite al autor utilizarla como parámetro para comparar y explicar diferencias en la capacidad productiva entre diferentes naciones.

*

John Stuart Mill ha ofrecido una respuesta que explica las variaciones encontradas en los índices de productividad entre naciones. Aparece un aspecto central, anterior a criterios vinculados a la industria (capacidad tecnológica, por ejemplo): no todas las naciones disponen del mismo acervo energético para sostener la industria.
El problema no está determinado por las circunstancias etnológicas que distinguen a «las razas», tampoco por las condiciones climatológicas que caracterizan a las diversas regiones geográficas. Ni siquiera es una condición ontológica la que determina a una raza como superior a otra en términos de capacidad física, intelectual, industrial, artística o militar. Al interior de una misma raza se pueden advertir diferencias importantes en términos de capacidad productiva. La diferencia radica en otro aspecto, mucho más central para la economía política liberal que piensa Mill.

La disposición de energía corporal para la actividad industrial determina el índice productivo de una nación. El problema principal no tiene que ver con su incremento, pues, incluso aquellas naciones cuyas gentes poseen abundante energía corporal, sobre todo en los climas cálidos, demuestran ser incapaces de apropiarse de sus beneficios industriales. La preocupación central con respecto al flujo energético no tiene que ver inicialmente con su caudal, sino con su cauce: ¿de qué modo es posible reorientar el curso de flujo energético de manera que propicie la dinamización de la producción industrial? ¿Qué formas de trabajo aplicado a los seres humanos son necesarias para que sea posible reorientar el curso de su energía corporal según el vector de optimización industrial? 
La clave antropológica de Mill viene dada por el deseo: las vías del goce marcan la pauta del devenir de la energía corporal. De este modo, el debido encauzamiento del flujo no se logra a través de la represión, la cual, imposibilitaría el despliegue energético e implicaría el estancamiento corporal, sino que resulta de una readecuación de los objetos hacia los cuales se dirige el goce. No se trata de una actividad centralizada del Estado, que vendría a imponer un modelo uniforme de deseo. Cada individuo debe llevar a cabo, a partir de las herramientas que la vida social pone a su disposición (educación), una apropiada administración de sí mismo, que le permita apropiarse de aquello más íntimo: su vitalidad, no solo para controlarla, sino para expandirla en forma de energía corporal. Los seres humanos, al ocuparse de sí mismos, realizan una importante contribución, no solo a sus propias vidas, sino a la vida de la raza, de la nación. Se trata de una moral que la economía política liberal ubica en la base de su proyecto de incremento en la productividad.

\section{Deus ex machina: religión, estrés y enmienda en John Stuart Mill}

Lo religioso, en Mill, participa de aquellos trabajos destinados a la captura de la energía vital de los seres humanos, con lo cual contribuye al proyecto productivo nacional. El propósito de esta breve consideración es identificar en Mill los modos de participación de lo religioso del ensanchamiento de la fuerza productiva nacional, así como valorar la continuidad de la propuesta de Mill con aquello que hemos caracterizado como teología económica.

Hemos establecido que la producción de energía industrial es el resultado de una serie de trabajos sobre los cuerpos de seres humanos, en procura de que cada individuo llegue a ser un administrador de su propia existencia. Así, uno de los principios de la economía política liberal, viene a participar de la tradición de teología económica cristiana, donde se solicitaba al cristiano ser un administrador de sí, como principio fundamental para mantener la salud del cuerpo místico. No obstante, resultaría inapropiado en este punto establecer que el proyecto liberal de Mill es un recipiente pasivo de los predicados de la tradición teológica, pues, tal como veremos, el filósofo inglés se distancia de algunos de sus principales predicados, en particular, aquello que vincula divina providencia y el uso del mal. De esta forma, pensar la naturaleza de la religión, un arcano problema al cual se aproxima Mill, solo tiene sentido para considerar los rastros de economía teológica al interior de su economía política liberal.

En este apartado me ocuparé de establecer cómo el abordaje de J. S. Mill sobre la naturaleza, la religión y la 
tradición cristiana ofrece una consideración sobre elementos centrales de la teología económica desarrollada por el cristianismo. La discusión sobre una divinidad que tiene una relación con su creación y criaturas, no solo de producción, sino, además, de gestión, donde incluso el mal (según lo determina la tradición de la divina providencia) debe ser dispuesto al objetivo de vigorizar y mejorar la vitalidad creada, es asumida por Mill y reintroducida dentro de los principios de economía política liberal. Nos interesa analizar cómo se resuelve el problema de la esencia religiosa, el vínculo del ser humano con respecto a su creador, el papel de la creencia, y de aquello que Mill denomina Religion of Humanity en relación con la sociedad liberal.

En sus ensayos sobre religión, ${ }^{4}$ John Stuart Mill (Mill, "Three Essays on Religion", 369-489) nos sugiere que el encuentro entre creador y criatura no muestra una divinidad cargada de gloria, mucho menos de una disposición docente, donde toma de la mano al ser humano, aún infante, y lo conduce entre una creación incólume. Según Mill, el primer aviso que el ser humano tiene de dios no viene de lo más alto, su voz no cae del cielo poderosa

4 Los dos primeros ensayos, Nature y Utility of Religion, fueron escritos entre 1850 y 1858 , el tercero, El Teísmo, entre 1868 y 1870. La publicación de estos manuscritos aconteció de modo póstumo. como un trueno. No hay parusía donde veamos al Señor de los cielos descender desde lo alto de una colina con su buena nueva.

A rastras, exhausto y abatido, un dios maltrecho apenas alcanza llamar la atención de su criatura. Extenuada, la deidad clama auxilio, según relata Mill en contra de toda una tradición apologética. Tras de sí, arrojado en el suelo, yace una divinidad rendida ante una empresa que le demostró impotente: erradicar el mal de su propia creación. Al lidiar con un material imbuido de decadencia, dios mismo ha sido infectado y ha caído fatigado. Su obra está incompleta y se sabe incapaz de llevarla a buen término. Dios no dona al ser humano su creación a modo de obsequio, sino por necesidad. Rendido, se ve en la obligación de ofrecer su proyecto a una criatura finita, imperfecta, portadora de la misma decadencia por subsanar: el mal debe ser desterrado, es la consigna principal. ${ }^{5}$

El primer ensayo sobre la religiónde John Stuart Mill antecede, por más de tres décadas, a la Gaya Ciencia, cuyo Requiem aeternam Deo (acápite ciento veinticinco) anunciaría la descomposición del cadáver divino (Nietzsche

5 Con esta postura, Mill se separa del núcleo central de la tradición apologética. Que la creación es obra de una divinidad potente y no un mero mecánico que trabaja con material ya dado, fue una discusión importante para los padres de la Iglesia, ver Athanasius (57). 
120). No resulta viable, sin embargo, asimilar ambos textos, al menos en sus conclusiones. Su tesis con respecto a la divinidad no admite la misma lectura, pues no se comunica la muerte de dios, ni el aviso, indispensable y festivo, de su exilio perpetuo. Tampoco se condena, como en Nietzsche, la hipocresía humana como un crimen mayor al deicidio, toda vez que se convive con significados ultramundanos por los cuales la divinidad sobrevive, incluso luego de fenecer, en la moral, la naturaleza, también la razón (Eagleton 151-173). Esta lectura no resulta posible en los ensayos del filósofo inglés, donde la deidad sigue presente junto a su creación.

Dios yace fatigado, no muerto. El proyecto de la creación ha llevado sus fuerzas al punto de fallo, pero no al fallecimiento. En los ensayos sobre religión, Mill no postula una vacante dejada por la divinidad, en torno a la cual el ser humano asume, jactancioso, el rol de amo del universo. El ensayo, La Naturaleza (1850), por ejemplo, no afirma que la deidad deja su proyecto a la deriva. Ciertamente le faltan fuerzas para llevar a buen puerto su empresa, pero no intención, pues anhela el triunfo del bien. Dios desea continuar, pero flaquea. De este modo, los templos no son mausoleos donde se deposita lamento o escarnio, ni están tibias sus paredes por el laudable sangrado, como llegará a anunciar el loco. A lo sumo hematidrosis. Procede explicarse la humedad por efecto del sudor divino: el creador está exhausto, fatigado. Ya el relato de la creación había advertido sobre la necesidad de descanso de la deidad al finalizar su obra.

Asimismo, dios no ofrece su proyecto a la criatura para desatender su creación. El filósofo inglés deja entrever una divinidad que, a pesar de sus buenas intenciones, experimenta un no poder más (Han, 2012), un rendimiento, en su acepción de falta de fuerzas que imposibilita concluir, producir o extraer utilidad. No se declara ni conmemora intención de abandono, de exilio.

Dios está rendido, ¿qué lo doblega? Su opresión no viene de una tiranía externa que le ha impuesto la carga de la creación, pues su soberanía es suprema. La deidad en Mill está obligada por su propio empeño, es decir, por una incapacidad de negar la obligación de su propio proyecto. La rendición divina proviene de un emprendimiento que llevó su fuerza al fallo, a la fatiga. En esta formulación, dios padece estrés (Sloterdijk, 2017) y requiere una asociación que le permita liberar la carga. La criatura será en adelante un asociado que viene a ensanchar la capacidad, el rendimiento divino. En un giro con respecto a los Padres de la Iglesia, la fatiga de dios suscita la asociación primera entre creador y criatura, que 
acontece para alivianar el estrés divino de una tarea altamente demandante: extirpar el mal.

El mito de la modernidad, según Mill, no narra la muerte de la deidad, en cuya vacante se posiciona el ser humano, quien sublimará lo celestial en instituciones y valores que, aunque secularizados, beben de predicados religiosos. El mito moderno anuncia, según el filósofo inglés, la primera comunidad del estrés. El núcleo mitológico de la modernidad comunica un emprendimiento conjunto, una asociación primordial entre divinidad y criatura que permite alivianar el estrés de las fibras que movilizan la empresa creacionista. La divinidad experimenta un no poder más, por lo que su criatura ofrece su aporte para inyectar capacidad. Se trata de una asociación primigenia que viene determinada por la tarea de enmendar lo creado, hasta que el bien se imponga a plenitud.

El liberalismo promete felicidad, toda vez que la carga de los emprendimientos esté distribuida en el cuerpo social, de tal forma que no se lleve al punto de fatiga alguna de sus fibras (al menos, aquellas que asuman con seriedad el contrato ciudadano), comenzando por las fibras del cuerpo glorioso de dios. En Mill tenemos una aproximación a la religión que la piensa a partir de niveles de estrés del cuerpo social. En su tratado sobre la naturaleza, el filósofo inglés no nos presenta una divinidad que agoniza o que deja la creación a su suerte. Tampoco el ser humano se presenta como el revelo triunfal, que asume la vacante en el trono universal. Se trata de una consideración de la primera asociación de emprendimiento, como una colaboración que procura inyectar capacidad al rendimiento divino y, a su vez, subsanar el estrés padecido por el emprendimiento creacionista. La escena primigenia de una sociedad del estrés se propone en la base de la sociedad liberal del siglo XIX.

$*$

¿Qué ha llevado a la divinidad a este punto de fatiga? Lo hemos dicho, la imbatibilidad del mal. Esta perspectiva se comprende y desarrolla a partir de un principio que articula los ensayos sobre religión de Mill, según el cual, el bien y el mal comparten una misma cualidad: se (re)generan a partir de sí mismos. Esta autogeneración es, por su mismo juego, negativa, en tanto la reproducción de uno significa la contracción de su opuesto. Del bien no se predica el mal y, correlativamente, el ensanchamiento del bien implica el repliegue del mal. Mill lee la parábola de los talentos (Mt 25:29) en esta clave, como una formulación antigua de este principio: el bien, también el mal, tiende a fructificar su propia clase en detrimento de su antítesis. Solo 
esta consideración permite demostrar, según el filósofo inglés, que la pobreza sea madre de tantos otros vicios. Del vicio, por el contrario, no predica ninguna virtud.

Resultaría contradictorio con este primer principio postular el uso providente del mal. J. S. Mill aborda el problema de la naturaleza, ese nombre colectivo para todas las cosas, actuales y posibles, a partir de su hipótesis de trabajo: "The only admisible moral Theory of Creationist is that the Principle of Good cannot at once and altogether subdue the power of evil". ${ }^{6} \mathrm{El}$ mal no puede ser tenido por mecanismo correctivo del vicio. Esto implica una ruptura con aquel criterio teológico (de estirpe estoica ${ }^{7}$ ) que explica el mal como estratagema en la concreción del mayor bien posible. De ser así, dios no solo permitiría la existencia del mal, lo querría, entonces, goza someter al ser humano al tortuoso e interminable camino a la virtud. Es una conclusión que Mill se niega a adoptar como principio de la naturaleza. Dios no desata el sufrimiento, lo padece. El carácter ortopédico del mal al interior del gobierno providente resulta falso $y$, como yerro, no debe ser adoptado o

6 (Mill, "Three Essays on Religion," 389). Esta perspectiva vuelve a aparecer en el segundo ensayo, "La utilidad de la religión", casi en términos idénticos (425).

7 Para ver los influjos entre la doctrina estoica y el cristianismo temprano ver Tuomas Rasimus, Troels Engberg-Pedersen, Ismo Dunderberg (2010). imitado por las costumbres y gobierno de los seres humanos.

Según el filósofo inglés, la creación exuda esta doctrina: el bien y el mal se derivan y multiplican a partir de sí mismos. Nunca uno sirve como agente del otro. De ahí que la naturaleza no debe ser tenida como norma del quehacer humano, pues de su acción se deriva también muerte, tortura y decadencia. En una tradición que Mill rastrea hasta Platón, la creación, si se la acepta como obra de un ser de bondad infinita, es el resultado de una divinidad que aportó todo cuanto tenía de bueno para ofrecer, pero tuvo que lidiar con un material intratable, constituido por una decadencia interna inextirpable:

One only form of belief in the supernatural -one only theory respecting the origin and government of the universe- stands wholly clear both of intellectual and moral obliquity. It is that which, resining irrevocably the idea of an omnipotent creator, regards Nature and Life not as the expression throughout of the moral character and purpose of the Deity, but as the product of a struggle between contriving goodness and an intractable material, as was believed by Plato, or a Principle of Evil, as was the doctrine of the Manicheans (Mill, "Three Essays on Religion”, 425).

Si se renuncia a la omnipotencia, la divinidad no puede querer el mal, 
ni siquiera como un recurso ortopédico, indeseado pero requerido. Dios se propone el bien, según se indica en los diálogos platónicos: "dios no es causa de todas las causas, sino sólo de las buenas" (Platón 140-141) Dios no es causa del mal. Para sus criaturas dispone el placer no el dolor: "the Creator desired the pleasure of his creatures" (Mill, "Three Essays on Religion", 457-458), nos dice Mill en su ensayo El Teísmo, pero, a pesar de su deseo, la divinidad no logra la plenitud del bienestar de lo creado. Desde esta perspectiva hay que renunciar a la omnipotencia divina, toda vez que el mal no se admite como intermediario del bien, es decir, dios mismo enfrenta limitaciones de rendimiento. La creación es un proyecto pendiente.

Luego, cualquier prosélito, independientemente de su credo, si este debe admitirse como verdadero, debería estar de acuerdo en un punto mínimo: "if Nature and Man are both the Works of a Being of perfect goodness, that Being intended Nature as a scheme to be amended, not imitated, by Man" (Mill, "Three Essays on Religion", 391). Dios no entregó la creación como espejo, donde el ser humano encontraría las vías de la perfección, por el contrario, ofrece un proyecto inacabado, una obra por finalizar. La única religión verdadera, en Mill, es aquella que alienta al ser humano a asumir su papel de colaborador de una divinidad que no puede más: la creación debe ser redimida, enmendada por la criatura.

$$
*
$$

El desarrollo de una teoría de la religión según la partenogénesis del bien (mal) establecida por J. S. Mill aporta una distinción con respecto a las corrientes de estudio de lo religioso del siglo XIX, principalmente las que estaban en boga en el medio académico británico de su época.

La naciente ciencia de la religión, principalmente aquella vinculada al clero a finales del siglo XVIII e inicios del XIX, adoptó una primera taxonomía cuaternaria, previo al gran giro que aportaría la gran ciencia del lenguaje al estudio de lo religioso, menos de un siglo después. El criterio divisorio asumía al cristianismo como la religión verdadera, y derivaba de ella una noción de religión que establecía el parámetro analítico: religión es la apropiada adoración de Dios, creador del cielo y de la Tierra. Las otras formas de religión que se admitían en los diccionarios de las religiones que circulaban en Gran Bretaña a inicios del siglo XIX, a saber, judía, islámica y pagana (esta última incluía un cúmulo diverso de "sectas", según el lenguaje de la época), se comprendían como formas más o menos alejadas de la adoración 
verdadera. ${ }^{8}$ No obstante, para Mill, la religión que se precia de verdadera no puede agotarse, ni caracterizarse principalmente, por la adoración de un dios vencido, que nos ha heredado una creación inacabada. No solo porque Mill, naturalista, evita un principio ultramundano, sino que, aún a pesar suyo, no sería la adoración el principio agente de lo religioso.

Previo al Iluminismo, los teólogos cristianos hicieron algo más que clasificar sectas en términos de una falsa o sucedánea adoración. Ahí donde encontraron el error idolátrico, se dieron a la tarea de explicitar las causas humanas que propiciaban la proliferación de esos rituales idolátricos meramente humanos. ${ }^{9}$ Estos estudios marcaron la base de lo que más tarde, durante la ilustración europea, y fuera de toda perspectiva proselitista, fue denominado el paradigma naturalista, que explicó lo religioso, no a partir de principios o referentes sobrenaturales, sino en términos de sus orígenes mundanos. Para el naturalista, la religión debía ser explicada a partir de sus causas naturales: humanas, sociales y ambientales. ${ }^{10}$

8 Masuzzwa (2005).

9 Es ejemplar de este modelo de explicación el texto de Josef de Acosta (2008 [1590]). Por varios siglos, el intento de Acosta de explicar las prácticas rituales y de adoración de los habitantes de las Nuevas Indias se adelantó en mucho a las teorías naturalistas que intentarían explicar lo religioso.

10 Para un estudio que se aproxima al paradigma naturalista desde uno de los autores más emblemáticos del siglo XIX, en lo que respecta al estudio de
El iluminismo británico desarrolló ampliamente la aproximación naturalista a lo religioso. A partir de una larga tradición filosófica, se vinculó el origen de lo religioso con un sentimiento en particular, el miedo: Primus in orbe Deos fecit timor. David Hume lo expresó en breve: "The primary religion of mankind arises chiefly from an anxious fear of future events". ${ }^{11} \mathrm{Se}$ gún este principio, el miedo es un sentimiento potente del cual ha emergido, entre otras creaciones humanas, lo religioso. A finales del siglo XIX, el célebre trabajo de Edward Tylor, Primitive Culture (1871), particularmente en lo que respecta a sus análisis del animismo, ritos y ceremonias de "los salvajes", expresiones del estadio infantil de la humanidad, consolidó la idea de que toda forma religiosa (que Tylor explica como sistemas de creencias) se vincula, en la temprana humanidad, con los distintos miedos ${ }^{12}$ que los seres humanos tienen con respecto a la vida y al porvenir.

No obstante su afinidad con la perspectiva naturalista, J. S. Mill advierte como falso el principio que explica lo religioso como resultado del

lo religioso, ver Andrew C. Dole, Schleiermacher on Religion and the Natural Order (2010).

11 David Hume, The Natural History of Religion (1889 [1757]).

12 Este principio de inteligibilidad se desarrolla ante todo en el segundo volumen del estudio de Edward Tylor, Primitive Culture. Researches into the Development of Mythology, Philosophy, Religion, Language, Art and Costume (1920). 
miedo, ${ }^{13}$ a pesar de considerarlo un sentimiento tan poderoso y universal, apropiado para el disciplinamiento. Para Mill, el origen de los dioses no tiene como base el temor a lo desconocido, con lo cual, no es de la ignorancia, propiamente, que se deriva lo religioso. De ahí la pregunta central en el Segundo ensayo de Mill: iwhat it is in human nature which causes it to require a religion; what wants of the human mind religion supplies, and what qualities it developes? ("Three Essays on Religion”, 418).

Para el filósofo inglés, la base antropológica de lo religioso, al igual que la poesía, tiene su anclaje en la imaginación humana en tanto se proyecta hacia un mismo anhelo: la posibilidad de acceder a una vida más hermosa y bondadosa. La religión se vincula al anhelo de felicidad: "So long as human life is insufficient to satisfy human aspirations, so long there be a craving for higher things, which finds its most obvious satisfaction in religion" (Mill, "Three Essays on Religion", 419).

13 También la crítica a asumir el miedo como fuente de lo religioso tiene una larga tradición y tuvo una importante recepción después de los trabajos de J. S. Mill. El famoso estudio sobre la religión semita, que tanto inspiró a Sigmund Freud, establece una crítica a esta forma de comprender lo religioso como derivado del miedo: "It is not with a vague fear of unknown powers, but with a loving reverence for known gods who are knit to their worshippers by strong bonds of kinship, that religion in the only true sense of the word begins. Religion in this sense is not the child of terror" (Smith 54-55).
Religión dice, en primer lugar, del reconocimiento del carácter incompleto y decadente de ambos, creación y criatura. En segundo lugar, la religión aparece como un saber imaginativo que, en la forma de un anhelo, propone a la criatura el horizonte de una vida más bella, bondadosa y feliz.

Sin lugar a dudas, del principio que ancla lo religioso al miedo se deriva una conclusión similar, con un alcance distinto, según la cual, la religión viene a ofrecer una realidad alternativa, más bella y placentera, donde nuestros miedos y angustias finalmente serán subsanados. En este sentido, lo religioso no se propone cancelar el mundo o mejorarlo, ni siquiera aceptarlo como bueno, sino soportarlo como pasajero. Luego, la afirmación de la religión como un anhelo de bondad y belleza se puede predicar de la religión como nacida del miedo y la angustia, al modo de un fármaco ${ }^{14}$ que calma nuestra ansiedad ante la existencia, de modo que sea llevadera, sin implicar un compromiso con el mundo.

Empero, J. S. Mill se separa de esta última consideración, como explicación del alcance genuino de la religión. La religión verdadera no consuela al

14 Fármaco, en el uso que se asigna a lo religioso según esta perspectiva, tendría que entenderse en el doble sentido que Derrida advierte en el texto platónico, como remedio y veneno (Derrida 140-175). 
ser humano, ni lo resigna a soportar las penas mundanas. La religión verdadera en Mill no es indistinta al mundo y su decadencia. Por el contrario, lo religioso expresa el ánimo para enmendar lo creado. Esto constituye, en Mill, la esencia de todo sentimiento religioso, aunque pueda ser vulgarizado.

Una religión coherente con las aspiraciones morales e intelectuales más altas, no ofrece su anhelo de felicidad al modo de un analgésico o calmante que nos consuela frente a la miseria, sino como mediante el requisito de tomar partido en un proyecto inconcluso. El filósofo inglés indica: "A virtuous human being assumes in this theory the exalted character of a fellow-labourer with the Highest, a fellow-combatant in the great strife; contributing his little, which by the aggregation of many like himself becomes much, towards that progressive ascendancy, and ultimately complete triumph of good over evil" (Mill, "Three Essays on Religion", 425). ${ }^{15} \mathrm{El}$ ser humano, desde esta perspectiva, deviene un colaborador con el Altísimo en la lucha que pretende el triunfo

15 Se trata de una idea que Mill yahabíadesarrolladoensuensayo anterior, La Naturaleza, referida a la divina Providencia: "if as is the more religious theory, Providence intends not all which happens, but only what is good, then indeed man has it in his power, by his voluntary actions, to aid the intentions of Providence; but he can only learn those intentions by considering what tends to promote the general good" (Mill, "Three Essays on Religion”, 397). último del bien sobre el mal. Dios no desaparece, empero, cansado, rendido, precisa de un socio que contribuya a su empresa: una religión es coherente cuando presenta al ser humano como el socio de una divinidad que no ha podido más.

El anhelo religioso no consuela, pues no está anclado en el miedo, por el contrario, induce a responder afirmativamente a la solicitud de una divinidad que no ha podido llevar a buen término su creación, y se dirige a su criatura en procura de asistencia. Luego, desde la vía religiosa, el ser humano es conducido a mejorar lo creado y aquello que tiene frente a sí mismo. La verdadera religión, en Mill, aporta las formas de imaginación y anhelo requeridas por la empresa que constituye la esencia misma de su economía política: enmendar la creación desde sus cimientos, llevarla al máximo de sus posibilidades.

$\mathrm{Al}$ arribar a este punto, se podría argumentar que la postura de J. S. Mill con respecto a la religión no avanza en lo que sería una consideración sobre la utilidad de la religión según ha sido formulada en torno al liberalismo. Resulta conocida la fórmula: la religión aporta al control moral de la población obrera, de modo que la conducta de los trabajadores se ajuste a los requerimientos disciplinarios de la productividad industrial. El aporte de la 
religión, si bien no se limita a resignar a las personas al sufrimiento de su existencia so pena de una ulterior compensación, ofrece seres humanos dóciles a la autoridad civil como representante terrenal de la soberanía divina.

Adam Smith, por ejemplo, en su consideración De los gastos de las instituciones para la instrucción de las gentes de todas las edades, se ocupa del financiamiento de instituciones eclesiásticas, ¿se deben o no financiar instituciones religiosas? Siguiendo a D. Hume, Smith indica que las Iglesias pueden ofrecer ventajas a los Estados: "ecclesiastic establishment, though commonly they arose at first from religious views, prove in the end advantageous to the political interest of society" (Smith, An Inquiry into the Nature and Causes of the Wealth of Nations, 796). La gran ventaja de la religión en esta materia, según Smith, se basa en la autoridad que impone mediante el miedo porque "the fears which it suggets conquer all other fears" (Smith, An Inquiry into the Nature and Causes of the Wealth of Nations, 796). Se trata del carácter útil de la religión, en particular de instituciones eclesiásticas, donde lo religioso sirve al Estado y a la industria debido al control moral de las clases obreras (claramente, esta posición de Adam Smith sobre las instituciones eclesiásticas no representa toda su comprensión sobre el peso de la teología cristiana en la conformación misma de una economía política, ${ }^{16}$ sin embargo, es común que literatura secundaria lo proponga como el total del lugar de lo religioso-teológico en el liberalismo).

J. S. Mill no asume que el aporte de la religión se limite o concentre en el control moral de las conductas (particularmente, de las clases trabajadoras). Esta consideración se deriva de las tesis que su maestro, Jeremy Bentham, habría formulado con respecto a la religión natural (Bentham, 1822). Bentham abordó las limitaciones de la religión para influir en el comportamiento de la población, en comparación con otros ámbitos de la cultura. Esto se explica a raíz de los mecanismos religiosos que procuran asegurar la sumisión, los cuales, por estar basados en el dolor y la represión, desatan una serie de daños colaterales a los feligreses: "The force, which thus casts into the already over poised scale of misery, may be best appreciated by stating, that it frequently drives the sufferer into insanity" (Bentham 72). La crítica de Bentham está dirigida a toda comprensión de lo religioso en términos de tácticas de poder estrictamente negativas. Si la influencia de lo religioso estuviera determinada solo por la represión y el dolor que procura la docilidad de temperamento, el daño sería mucho mayor que el beneficio, toda vez que de lo religioso se derivarían padecimientos psicológicos, según Bentham.

$\overline{16 \text { (Pimentel, 2014). }}$ 
El padecimiento religious anxiety se le antojaba como una prueba fehaciente de esta condición.

Mill retoma el marco analítico más amplio de Bentham, y desarrolla una crítica del componente moralizador/represivo de lo religioso, toda vez que es tenido como el aporte fundamental de lo religioso a la sociedad liberal:

Belief, then, in the supernatural, great as are the services which it rendered in the early stages of human development, cannot be considered to be any longer required, either for enabling us to know what is right and wrong in social morality or for supplying us with motives to do right and to abstain from wrong. Such belief, therefore, is not necessary for social purposes at least in the coarse way in which these can be considered apart from the character of the individual human being [...] If supernatural beliefs are indeed necessary to the perfection of the individual character, they are necessary also to the highest excellence in social conduct: necessary in a far higher sense than the vulgar one, which constitutes it the great support of morality in common eyes (Mill, "Three Essays on Religion", 417).

En este sentido, John Stuart Mill procura apartarse de lo que considera una valoración vulgar del papel religioso cuando se le reduce a ser un bastión del Estado, en la forma de un mecanismo de control moral de la población. El mayor aporte de lo religioso a la sociedad liberal no se agota, solo en un nivel superficial, aunque más evidente, en el despliegue de formas de represión de las pasiones y la conducta de las clases obreras. La esencia de la religión no dice represión, ni siquiera en términos de la mayor utilidad que puede ofrecer a la sociedad liberal.

*

Volvamos a la pregunta de Mill: iwhat it is in human nature which causes it to require a religion; what wants of the human mind religion supplies, and what qualities it developes? ("Three Essays on Religion", 418). La respuesta viene dada por aquello que el filósofo inglés denomina la esencia de la religión: "The best persons have always held it to be the essence of religion, that the paramount duty of man upon earth is to amend himself: but all except monkish quietists have annexed to this in their inmost minds [...] the additional religious duty of amending the world, and not solely the human part of it, buy the material; the order of physical nature" (Mill, "Three Essays on Religion", 383). Lo religioso, en la explicación naturalista de Mill, responde a un deseo del ser humano: enmendarse a sí mismo, superarse, mejorarse. Por lo tanto, se vincula con el desarrollo de todas las cualidades que contribuyan a satisfacer este deseo con respecto a 
sí mismo y que se proyecta al resto de la creación. Luego, la productividad de una nación, según los principios de economía política, encuentran su raíz antropológica en esta misma esencia religiosa, a saber, el deseo de enmendarse a sí mismo.

Mill utiliza el verbo to amend (enmendar), toda vez que se refiere a la esencia de la religión, a la raíz antropológica de todo fenómeno religioso. A pesar de que el filósofo inglés es lo suficientemente cuidadoso como para emplear conjugaciones de este verbo cuando se refiere a la esencia de lo religioso, la traducción al español emplea constantemente el término corregir, ${ }^{17}$ como si fuera un sinónimo apropiado. Sin embargo, leído desde el contexto de los tres ensayos sobre religión, este cambio desplaza gran parte del sentido que nos propone Mill con enmendar.

Enmendar, tanto en inglés como en español, se deriva del latín, que combina el prefijo privativo ex, fuera, y el término mendum, falta, error, defecto. Este concepto se deriva de la idea de extraer el defecto en algo, aplicar una acción que anule o subsane el error. Enmendar, según su etimología,

17 En una de las citas que hemos empleado más arriba se torna evidente el cambio del "to be amended" de Mill: "si la Naturaleza y el Hombre son ambos la obra de un Ser de bondad perfecta, ese Ser concibió la Naturaleza como un plan para ser corregido por el Hombre, no imitado" (John Stuart Mill, Tres ensayos sobre religión, 78). no presupone un estado previo perfecto, puro, inmaculado que requiere retomarse, sino, propiciar el bienestar toda vez que resulta eclipsado. De ahí que corregir, como un enderezar la dirección, no recoge el contexto en el cual se nos propone esta idea. No se enmienda para volver o retomar un rumbo perdido, sino para propiciar algo no (necesariamente) acontecido. ${ }^{18}$ Cobra sentido el uso del término en Mill, a partir del contexto de enunciación: no se enmienda una creación/criatura cuya bondad primigenia debe ser restituida, enmendar comunica la posibilidad de lo no acaecido, el triunfo definitivo del bien, que la divinidad, en el principio, no fue capaz de instituir. Enmendar dice de una tarea proyectiva, no retroactiva, y aún pendiente. No es un retorno, sino un arribo a lo insólito.

La religión, en la perspectiva de J. S. Mill, se vincula con esta necesidad antropológica por la cual el ser humano desea introducir en la historia aquello no acaecido: el triunfo definitivo del bien. Lo religioso se deriva de un deseo/aspiración de enmendar lo creado, no para devolverlo a la bondad perdida, que nunca tuvo, sino para

18 Debemos pensar enmendar en el sentido que Heidegger presenta "el estado de caída" del "ser ahî": "tampoco debe tomarse, por ende, como caída desde un "estado primitivo" más alto y puro. De esto no solo no tenemos ónticamente experiencia alguna, sino tampoco ontológicamente posibilidades ni hilos conductores de exégesis" (Heidegger 195). 
conquistarla finalmente. De esto se desprende la imaginación religiosa, no por la necesidad de consuelo, sino del impulso de la criatura por hacer algo mejor de sí mismo. El deber inventivo, más que ortopédico, que el ser humano tiene respecto de sí, se proyecta hacia todo lo creado, de modo que toda la creación sea enmendada de la decadencia y el mal que padece.

El compromiso hondo con esta tarea permitiría que cualquier credo, comprometido con esta consigna, coincida con aquello que Mill designa Religion of Humanity, una religión que no descansa en ninguna creencia: "a religion may exist without belief in a God, and that a religión without a God may be, even to Christians, an instructive profitable object of contemplation" (John Stuart Mill, "Auguste Comte and Positivism", 332). Más allá de posicionarse sobre la creencia, esta forma ideal de religión la piensa Mill en relación con una práctica de sí por la cual todo debe ser enmendado, llevado hasta sus máximas posibilidades. De esta forma, no solo las religiones particulares pueden brindar utilidad al proyecto liberal, sino que un desarrollo adecuado de la economía política, permite que las religiones mismas se eleven sobre sus formas «vulgarizadas». Es un beneficio en dos vías que se da cuando el ser humano, por las vías de una industrialización civilizada, hace de sí mismo un objeto digno de contemplación.
Debido a que el ser humano no puede, por imitación de la naturaleza, enmendarse a sí mismo y a la creación, debe propiciar aquello que no ha tenido lugar en la historia. Lo artificial ingresa en la consideración sobre lo religioso. Dios confía su proyecto a la criatura, de modo que lleve a buen término aquello que no pudo concretarse. La criatura, heredera de este proyecto, requiere de un suplemento que permita el triunfo definitivo del bien: "It is only in a highly artificialized condition of human nature that the notion grew up, or, I believe, ever could have grown up, that goodness was natural: because only after a long course of artificial education did good sentiments become so habitual, and so predominant over bad, as to arise unprompted when occasion called for them" (Mill, "Three Essays on Religion", 417). Mill refiere a un ejemplo: la limpieza. Nada más artificial que la limpieza y, sin embargo, el ser humano puede adquirir un amor por ella, ya que se inscribe dentro de su anhelo de bondad y bienestar.

Enmendar la obra de la divinidad requiere de lo artificial en sí mismo, nunca acaecido: Deus ex machina, algo debe ser introducido desde un afuera de la narrativa natural para enmendar la trama de la creación. No se puede confiar la expulsión del mal al instinto como reflejo de la naturaleza. En los ensayos de John Stuart Mill sobre la religión, la imaginación religiosa es 
una manifestación del principio antropológico básico, según el cual, el ser humano debe, por la vía del artificio, hacer de sí y de lo que lo rodea, algo mejor, que permita redimir la materia de toda decadencia.

Con esta última alusión a la necesidad de lo artificial como mecanismo para enmendar lo creado, que Mill introduce en sus consideraciones sobre la naturaleza y la religión, se enmarca el universo de acción básico de la economía política liberal: enmendar lo creado, primeramente, mediante la apropiada administración de sí, por medio de la cual, la criatura deviene una obra digna de contemplación, que, al ensanchar su vida, embellece y engranda la vida del cuerpo social, la nación. Pero, más aún, es la creación en su conjunto la que se enmienda por este mismo impulso. De ahí que la religión verdadera, según Mill, aquella que se expresa como el emprendimiento conjunto entre creador y criatura para enmendar el inconcluso proyecto creacionista, muestra el trasfondo natural que expresa o promete cumplir la economía política con respecto al progreso industrial de una nación. Incluso el recurso a lo artificial, por lo cual, la criatura se enmienda a sí misma, indica en qué medida todo recurso de factura humana, por ejemplo, los empeños de J. P. Joule en relación con el motor, participa de la honda expresión humana por enmendar lo creado hasta el punto donde el bien se imponga plenamente sobre el mal.

\section{Conclusión}

La presencia (incómoda) del fundamentalismo religioso, ${ }^{19}$ en sus distintas manifestaciones, alcances y solicitudes, ha obligado a reconsiderar el lugar de lo religioso en el escenario político mundial. Un énfasis sobre el carácter secularizado del mundo moderno auguró, prematuramente, la muerte de toda forma religiosa, o bien, su transformación en modelos no heterónomos de experiencia humana, siempre y cuando se limiten a la esfera privada. La presencia pública de lo religioso solo sería explicada (permitida) en términos utilitarios, como policía moral, por ejemplo, so pena de acatar con silencio los dictados del mundo moderno. Esta versión no aportó un panorama equívoco del mundo moderno, pero, sin duda, se trata de una visión incompleta, pues, lejos de morir, o replegarse en el ámbito privado, solicitudes ancladas en creencias religiosas son inscritas en el ámbito público, en ocasiones, con recurso a la violencia.

Si bien un aviso sensato nos llama a leer esta primavera religiosa en el ámbito público como un efecto de contragolpe del modelo socioeconómico regente, no debemos caer en la

19 Armstrong (2001). 
tentación de leer estos acontecimientos con las premisas analíticas derivadas del augurado exilio de lo religioso al ámbito de la vida privada, etapa previa a su final desaparición. Antes que una lectura sobre los usos que el neoliberalismo hace de los fundamentalismos religiosos, conviene sin duda repasar los fundamentos teológicos que soportaron el despliegue de la economía política de tipo liberal, por medio del cual, la tradición cristiana no fue abandonada por la modernidad, por el contrario, se le asumió en profundidad.

Según hemos considerado atrás, en la obra de John Stuart Mill, retorna una pregunta de raigambre teológica: ¿cuáles son las condiciones, los ejercicios y la economización (disposición, uso, orden) que cada uno debe cumplir de modo que se complete el plan (inconcluso) de Dios? O bien, ia partir de cuáles prácticas corporales individuales se asegura la inmunización y vigorización de la nación? La respuesta que ofrece el filósofo londinense consiste en ahondar la economía política de la carne por la cual, a partir del uso adecuado que cada quien haga de sí, se logra la salud del cuerpo social, y así, la promesa del crecimiento de todo bien. De esta forma, el proyecto liberal no se presenta como el corte entre una edad moderna secularizada frente a un antiguo régimen tributario de lo religioso, en su lugar, se ahonda una cuestión central en la teología económica, referida a las prácticas económicas de sí que aseguran la salud del cuerpo mayor.

El núcleo básico del Political Economy de Mill profundiza, en este particular, el legado teológico que el cristiano ofrece al mundo moderno: el ser humano debe enmendarse, y junto a él, debe enmendar todo lo creado. El derrotero viene dado por una apropiada administración de sí. El progreso se mide por nuestra aproximación a un estado no acaecido: el triunfo perpetuo del bien sobre el mal, donde cada uno, y el conjunto al cual pertenecemos, seremos capaces de ofrecer el mayor rendimiento como respuesta al don que hemos recibido del cuerpo social. En Mill, el contrato que establecemos con el creador participa de una teología de la gracia.

La utilidad de la religión, por parte del liberalismo, admite una lectura distinta a la policía moral. En el mito moderno, donde J. S. Mill habla de la primera sociedad contractual entre divinidad y criatura, para alivianar el estrés de una empresa altamente demandante, lo religioso se muestra, simultáneamente, como repliegue y expansión del nudo antropológico básico que define a la humanidad: lo creado debe ser enmendado. Solo desde esta perspectiva, lo religioso ofrece su más profundo y verdadero aporte a una sociedad liberal, en tanto ayuda al 
ensanchamiento de la energía e imaginación que requiere el nuevo orden: que cada uno haga de sí algo mejor de lo que es. La captura de vitalidad por parte de la economía política liberal implica que la vida, amenazada desde el interior, requiere ser enmendada, esto es, nutrida, extendida, ensanchada, mejorada, solo entonces el progreso industrial resulta empíricamente factible. Se trata de una tarea que debe ser realizada desde varios frentes, entre ellos, desde la vía religiosa.

De esta forma, la teoría de la religión natural se encuentra con una teología económica cristiana, según la hemos desarrollado en otro lugar. En otro registro lingüístico, el trabajo de J. S. Mill sobre la religión se inscribe dentro de la tradición teológica cristiana, cuando deriva del encuentro creador-criatura una solicitud básica: que el ser humano devenga un ecónomo, un eficiente administrador de sí que vela por el ensanchamiento del bienestar. Encarado a su creador, el ser humano descubre la tarea que recae sobre sus hombros: mejorar su condición, no solo a nivel espiritual, sino material.

Cuando nos preguntamos al inicio, a partir de los Principles de John Stuart Mill, ¿cuáles son los antecedentes históricos que han permitido a la economía política liberal pensar, en términos de una administración individual de la propia vitalidad, los problemas básicos con respecto al gobierno de los seres humanos requeridos por su proyecto, ante todo, aquella parte que se delega a los individuos mismo? La herencia de la teología cristiana es profunda. Resulta oportuno indicar que, incluso la categoría central de la economía política moderna, vida, más que remitir a un concepto enteramente biológico o médico, corresponde al ahondamiento de una teología de la carne, del exceso de vitalidad (que reúne ambos, vulnerabilidad y potencia/energía), a partir del cual el pensamiento económico de la modernidad capturó el nuevo horizonte abierto por la biología y el desarrollo de las ciencias médicas y del hombre.

\section{Referencias bibliográficas}

Armstrong, Karen. The Battle for God. New York: The Random House, 2001. Impreso.

Bentham, Jeremy. Analysis of the Influence of Natural Religion on the Temporal Happiness of Mankind. London: R. Carlile, 1822. Consultado el 26 de setiembre de 2017: https://ia601408.us.archive.org/8/items/analysisinfluenOobentgoog/analysisinfluenOobentgoog.pdf.

De Acosta, Josef. Historia natural y moral de las Indias. Editado por Fermín Pino-Díaz. Madrid: Consejo Superior de Investigaciones Científicas, 2008 [1590]. Impreso. 
Derrida, Jacques. La diseminación. Traducido por José Arancibia. Madrid: Fundamentos, 2015 [1972]. Impreso.

Dole, Andrew. Schleiermacher on Religion and the Natural Order. New York: Oxford University Press, 2010. Impreso.

Eagleton, Terry. Culture and the Death of God. New Haven: Yale University Press, 2014.

Freud, Sigmund. Obras Completas. Vol. 21. Traducido por José Luis Etcheverry. Buenos Aires: Amorrortu, 1992. Impreso.

Han, Byung-Chul. La sociedad del cansancio. Traducido por ArantzazuSaratxaga Arregi. Barcelona: Herder, 2012. Impreso.

Hardy, Edward, ed. The Library of Christian Classics: Christology of the Later Fathers. Vol. III. Philadelphia: The Westminster Press, 1954. Impreso.

Heidegger, Martín. El ser y el tiempo. Traducido por José Gaos. México: FCE, 2005 [1927].

Hume, David. The Natural History of Religion. Foreword by John M. Robertson. London: A. and $\mathrm{H}$. Bradlaugh Bonner, 1889 [1757]. Consultado el 24 de octubre de 2017: http://oll.libertyfund.org/ titles/340. En línea.

Hume, David. The Philosophical Works of David Hume. Including all the Essays, and exhibiting the more important Alterations and
Corrections in the successive Editions by the Author. Vol. 3. Edinburgh: Adam Black and William Tait, 1826. Consultado el 10 de octubre de 2017: http:// oll.libertyfund.org/titles/1483. Enlínea.

Masuza, Tomoko. The Invention of World Religions, or, How European Universalism Was Preserved in the Language of Pluralism. Chicago: The University of Chicago Press, 2005. Impreso.

Mill, John. The Collected Works of John Stuart Mill: Essays on Politics and Society. Vol. 18. Toronto: University of Toronto Press, 1977. Impreso.

Mill, John. The Collected Works of John Stuart Mill: Essays on Ethics, Religion and Society. Vol. 10. Toronto: University of Toronto Press, 2006. Impreso.

Mill, John. The Collected Works of John Stuart Mill: Principles of Political Economy with Some of Their Applications to Social Philosophy. Vol. 2. Indianapolis: Liberty Fund, 2006. Impreso.

Mill, John. Tres ensayos sobre religión. Traducido por Gerardo López Sastre. Madrid: Trotta, 2014 [1874]. Impreso.

Nietzsche, Friedrich. The Gay Science. Edited by Bernard Williams. Translated by Josefine Nauckhoff. Cambridge, UK: Cambridge University Press, 2008. Impreso. 
Pimentel, Jonathan. Economy of the Flesh: Nature and Economy in David Hume and Adam Smith. Tesis de Doctorado en Teología. Chicago: The Lutheran School of Theology, 2014. Impreso.

Platón. Diálogos V. República. Traducido por Conrado Eggres Lan. Madrid: Gredos, 1988.

Platón. Diálogos VI. Filbeo, Timeo, Critias. Traducido por Ángeles Durán y Francisco Lisi. Madrid: Gredos, 1992. Impreso.

Rasimus, Tuomas et al. Stoicism in Early Christianity. Grand Rapids, Michigan: Baker Academic, 2010. Impreso.

Rousseau, Jean. Ensayo sobre el origen de las lenguas. Traducido por Adolfo Castañón. México: Fondo de Cultura Económica, 1984. Impreso.
Sloterdijk, Peter. Estrés y libertad. Traducido por Paula Kuffer. Buenos Aires: EGodot, 2017. Impreso.

Smith, Adam. An Inquiry into the Nature and Causes of the Wealth of Nations. Eds. R. Campbell y A. Skinner. Indianapolis: Liberty Fund, 1981 [1776]. Impreso.

Smith, Adam. The Theory of Moral Sentiments (The Glasgow edition of the works and correspondence of Adam Smith). Edited byD. Raphael and A. L. Macfie. Indianapolis: Liberty Fund, 1982 [1759]. Impreso.

Smith, William. Religion of the Semites. New Jersey: Transaction Publishers, 2005 [1894]. Impreso.

Tylor, Edward. Primitive Culture. Researches into the Development of Mythology, Philosophy, Religion, Language, Art and Costume. Vol. I \& II. London: John Murray, 1920. Impreso. 
